\title{
Soliton Lattices in the Incommensurate Spin-Peierls Phase: Local Distortions and Magnetizations
}

\author{
Götz S. Uhrig ${ }^{1}$, Friedhelm Schönfeld ${ }^{1}$, Jean-Paul Boucher ${ }^{2}$ and Mladen Horvatić ${ }^{3}$ \\ ${ }^{1}$ Institut für Theoretische Physik, Universität zu Köln, Zülpicher Str. 77, Köln 50937, Germany \\ ${ }^{2}$ Laboratoire de Spectrométrie Physique, Université J. Fourier Grenoble I BP 87, \\ F-38402 Saint-Martin d'Hères Cedex, France \\ ${ }^{3}$ Grenoble High Magnetic Field Laboratory, CNRS and MPI-FKF, BP 166, 38042 Grenoble Cedex 09, France
}

(April 6, 2018)

It is shown that nonadiabatic fluctuations of the soliton lattice in the spin-Peierls system $\mathrm{CuGeO}_{3}$ lead to an important reduction of the NMR line widths. These fluctuations are the zero-point motion of the massless phasonic excitations. Furthermore, we show that the discrepancy of X-ray and NMR soliton widths can be understood as the difference between a distortive and a magnetic width. Their ratio is controlled by the frustration of the spin system. By this work, theoretical and experimental results can be reconciled in two important points.

75.10.Jm, 75.30.Fv, 75.50.Ee, 75.30.Kz

\section{INTRODUCTION}

Already 20 years ago, the physics around the spinPeierls transition fascinated many researchers (for a review, see Ref. [1]). In particular the incommensurably modulated I phase attracted much interest (e.g. [2 13]). Yet detailed experimental investigations of the nature of this phase were not possible at that time. The first spinPeierls transition in an inorganic compound, $\mathrm{CuGeO}_{3}$, was found only five years ago [14]. This made a multitude of experimental investigations possible (for a review, see Ref. [15]).

In particular, direct X-ray experiments in the I phase were performed by Kiryukhin and Keimer which permitted for the first time to detect the incommensurability of the distortion in $k$-space [16]. Even more, it was possible to look at the structure of the soliton lattice modulation by measuring the intensity of the third harmonic 17,18 .

On the other side, Fagot-Revurat et al. 19] were able to measure the distribution of local magnetizations in $\mathrm{CuGeO}_{3}$ in a beautiful NMR experiment. In a refined version it was now possible to deduce from such results the shape and the amplitude of the magnetic part of a soliton [20,21]. Three discrepancies to the conventional theories became apparent. The first concerns the amplitudes of the local magnetizations which is experimentally found to be much lower (factor 4 to 6) than predicted. Second, the X-ray soliton width $(13.6 \pm 0.3)$ is appreciably larger than the NMR soliton width ranging from 6 to 10 . Third, the widths are all larger than the ones theoretically predicted.
In the present work we will solve the first two discrepancies and argue with Zang et al. 22 that the remaining problems are connected to the neglect of interchain couplings.

To fix the diction let us state that we use the term soliton for the combination of a zero in the modulated distortion and the concomitant localized, bound spinon [4.52.23]. The distortive soliton width is the width of the kink-like zero of the modulated distortions. The magnetic soliton width is the spatial width of the local magnetizations [4, 5, 24, 23]. The incommensurate modulation in the I phase is viewed as an equidistant array (lattice) of solitons.

The paper is set up as follows. In section II we discuss the fact that the NMR line widths are much smaller than the theoretical ones and give an explanation for it in terms of the zero-point motion of the soliton lattice. Direct numerical calculations based on DMRG are shown in section III. They permit to address the second main point, namely the discrepancy between the soliton widths as measured by X-ray and by NMR. In section IV a detailed comparison to recent experimental data [20,21] will be presented. The concluding section contains a discussion of the open questions, namely the role of interchain couplings, and a summary of our results.

\section{AVERAGING DUE TO PHASONS}

In Ref. 19] the results were interpreted by fitting them to a Hartree-Fock theory of Fujita and Machida [12]. Fujita and Machida did not take into account that the expectation values which occur in the Hartree-Fock self-consistency problem become non-uniform in a nonuniform phase [25]. Thus they missed the important point that the local magnetizations $m_{i}:=\left\langle S_{i}^{z}\right\rangle$ are so strongly alternating that they are even antiparallel on every second site to the applied external magnetic field. The amplitude of the alternating component is strongly enhanced compared to the XY model as was predicted in a number of investigations $13,25,27,23]$.

Theoretically, there is no doubt that the spin-isotropic model has to be used to describe cuprate systems. Experimentally, however, the amplitude of an XY model fits much better than the enhanced amplitude of the isotropic XYZ model. The discrepancy can be explained by the 
fluctuations of soliton lattice which are induced by the presence of the so-called phasons [25].

The importance of zero-point motion of the crystal lattice in Peierls systems was already noted by McKenzie and Wilkins [28]. In the present work we will focus on the zero-point motion of the phasons. The phasons are very similar to phonons. If the deviation from commensurability of the wave vector characterizing the modulation $d:=|q-\pi|$ is small (low soliton concentration) and the soliton width $\xi$ is large a continuum approach can be used [2 13]. In this approach the discreteness of the underlying lattice does not appear. Thus the continuum Hamiltonian is invariant under continuous translations along the chains. The incommensurate modulation breaks this continuous symmetry giving rise to Goldstone bosons, the so-called phasons [29]. They refer to oscillations of the solitons about the equilibrium positions in their lattice just as phonons refer to oscillations of the atoms about the equilibrium positions in the crystal lattice. There is one important difference between phonons and phasons. There exist in general three phonon branches (2 transversal, 1 longitudinal) corresponding to the three spatial dimensions into which an atom can be moved. But there is only one phason branch since the modulation can only be moved along the chains. Note that this does not concern the fact that the phasons have a nondegenerate dispersion $\omega(\vec{k})$ which depends on a three dimensional vector $\vec{k}$. Like phonons the dispersion is linear in $\vec{k}$ for small values of $\vec{k}$, i.e.

$$
\omega^{2}=\left(c_{x} k_{x}^{2}+c_{y} k_{y}^{2}+2\left|c_{z}\right| k_{z}^{2}\right) / \rho
$$

in the notation of Ref. [29]. Hence, the phasons give rise to a $T^{3}$ contribution in the specific heat [29] which is indeed experimentally observed [30].

Before we proceed further we discuss briefly the effect of pinning. First we like to emphasize that there are two possible sources of pinning. The first one is pinning to the discrete lattice structure. The second is pinning to defects.

The first mechanism enters since the continuous translational invariance along the chains is given only in the continuum treatment which represents a certain approximation. So the phasons are only quasi Goldstone bosons of a quasi continuous symmetry breaking. Yet treating the incommensurate modulations as continuously translational invariant, i.e. shifting them without energy cost, is an excellent approximation if the solitonic width $\xi$ is not too small. In the course of our previous calculations 24] we noted that the energy difference between a modulation with the zero on a site and a modulation with the zero between two sites is of the order $J \exp (-C \xi)$ where $C$ is some constant of the order of the inverse lattice constant $c^{-1}$ in chain direction. Hence, for $\xi \approx 10 c$ this energy difference becomes negligibly small.

From the experimental point of view we come to the same conclusion. If there were a pinning of the modulation to the lattice structure the modulation would be commensurate with a period $L c$ where $L$ is an integer. This would imply that at most $L$ discrete local magnetization values $m_{i}$ occur. The experimental resolution, however, is sufficiently high to exclude this scenario since not a number of isolated peaks but a continuous distribution is observed in the NMR response [20,21].

The second mechanism is pinning to defects which break the translational invariance. Such an effect is certainly present but it is negligibly small in the pure samples. To obtain an estimate we argue that the defect concentration $x$ corresponds to a typical distance between two defects of $l=c / x$ where $c$ is the lattice constant in chain direction. This means that phasons with a wave vector below $k_{z} \approx 2 \pi / l=2 \pi x / c$ do not exist. This can be viewed as the effect of a gap $\Delta_{\text {pin }}$ induced by defect pinning. From eq. (11) we obtain the estimate

$$
\begin{aligned}
\Delta_{\text {pin }} & =\sqrt{\frac{2 c_{z}}{\rho}} \frac{2 \pi x}{c} \\
& =\Delta_{\operatorname{trip}} 2 \pi \sqrt{2} \frac{\xi_{0 z}}{c} x \\
& =500 \mathrm{~K} x
\end{aligned}
$$

where we used $c_{z}^{2}=u_{0} \xi_{0 z}^{2}, \Delta_{\text {trip }}=\hbar \sqrt{u_{0} / \rho}=24 \mathrm{~K}$, $\xi_{0 z}=0.69 \mathrm{~nm}$ [29] and $c=0.294 \mathrm{~nm}$ [31]. An upper bound for the defect concentration $x$ in the pure samples investigated is $10^{-3}$. So the defect pinning gap $\Delta_{\text {pin }}$ is lower than $0.5 \mathrm{~K}$. We conclude that defect pinning will become important only below $T \approx 0.5 \mathrm{~K}$ and does not need to be considered here.

The soliton comprises a zero of the modulated distortions and a spinon bound to this zero [23]. If there is a zero-point motion of the phasons this implies a certain motion of both the lattice distortions and the magnetic structure. It is plausible to assume that a certain averaging occurs which reduces the amplitude of the alternating magnetizations. This idea was first introduced in Ref. [25] to explain the difference between observed and computed magnetization pattern. (Note that Kiryukhin et al. 18 discussed a certain phasonic averaging linked to defects. Our approach does not rely on defects.) Here we present the detailed calculation and further estimates.

Let us assume that the local magnetizations $m_{i}$ can be described by two smoothly varying functions $a(r)$ and $u(r)$ which provide the alternating and the nonalternating component, respectively

$$
m_{i}=a\left(r_{i}\right) \cos \left(\pi r_{i}\right)+u\left(r_{i}\right)
$$

where we set the lattice constant to unity and $r_{i}$ denotes the component along the chains. The continuum approach results [12,22] are in fact of the form (5). Eq. (5) is the adiabatic result describing the completely static situation without phasons. Let us introduce now the phase variable $\hat{\Theta}\left(\vec{r}_{i}\right)$ where we use the hat to indicate that it is an operator as is the position of a harmonic oscillator. Thus (5) becomes

$$
\hat{m}_{i}=a\left(r_{i}\right) \cos \left(\pi r_{i}+\hat{\Theta}\left(\vec{r}_{i}\right)\right)+u\left(r_{i}\right) .
$$


In principle, the shift $\hat{\Theta}\left(\vec{r}_{i}\right)$ has to be inserted in the functions $a\left(r_{i}\right)$ and $u\left(r_{i}\right)$ as well. But these functions are slowly varying so that the influence of the shift on them is negligible. Assuming furthermore that the NMR experiments measure on a relatively long time scale we conclude that the local magnetization $m_{i}^{\exp }$ seen in experiment is simply the expectation value

$$
m_{i}^{\exp }=\left\langle\hat{m}_{i}\right\rangle
$$

In the harmonic approximation the phase fluctuations are interactionless bosons and the operators $\hat{\Theta}\left(\vec{r}_{i}\right)$ are linear combinations of the bosonic creation and annihilation operators. Then it is straightforward to compute the expectation value of the cosine

$$
\begin{aligned}
\left\langle\cos \left(\pi r_{i}+\hat{\Theta}\left(\vec{r}_{i}\right)\right)\right\rangle & =\cos \left(\pi r_{i}\right)\left\langle\cos \left(\hat{\Theta}\left(\vec{r}_{i}\right)\right)\right\rangle \\
& =\cos \left(\pi r_{i}\right) \exp \left(-\left\langle\hat{\Theta}^{2}\left(\vec{r}_{i}\right)\right\rangle / 2\right) .
\end{aligned}
$$

Since the dominant fluctuations are those at long wave lengths the dependence of $\left\langle\hat{\Theta}^{2}\left(\vec{r}_{i}\right)\right\rangle$ on the site index $i$ should not be important. Hence we introduce

$$
\gamma^{\prime}:=\exp \left(-\frac{1}{2 N} \sum_{i}\left\langle\hat{\Theta}^{2}\left(\vec{r}_{i}\right)\right\rangle\right)<1
$$

where $N$ denotes the number of sites in one chain. The reduction factor $\gamma^{\prime}$ is similar to a Debye-Waller factor which accounts for the non-vanishing atomic motion due to phonons. It reduces the amplitude of the alternating component only. From eqs. (6, $6,8,9)$ we find

$$
m_{i}^{\exp }=\gamma^{\prime} a\left(r_{i}\right) \cos \left(\pi r_{i}\right)+u\left(r_{i}\right)
$$

where the essential amendment compared to (5) is the reduction of the alternating component by $\gamma^{\prime}$. It is plausible to explain the discrepancy between experimental and adiabatic theoretical amplitude by the zero-point motion of the phasons which leads to a finite value of $\left\langle\hat{\Theta}^{2}\left(\vec{r}_{i}\right)\right\rangle$ and hence to $\gamma^{\prime}<1$. We will present further support for this idea in section IV.

Before turning to estimates for $\gamma^{\prime}$ we point out how one can take the reduction $\gamma^{\prime}$ into account if the result of the adiabatic calculation is not given in the form (5) but as a set of discrete values $\left\{m_{i}\right\}$. This is the case for any direct adiabatic numerical treatment (see, e.g., section III) which does not use the continuum approach. Then one has to deduce in a first step estimates for the slowly varying functions $a\left(r_{i}\right)$ and $u\left(r_{i}\right)$ from the $m_{i}$. The most natural way to do this is by taking local averages

$$
\begin{aligned}
& a\left(r_{i}\right)=m_{i} / 2-\left(m_{i-1}+m_{i+1}\right) / 4 \\
& u\left(r_{i}\right)=m_{i} / 2+\left(m_{i-1}+m_{i+1}\right) / 4 .
\end{aligned}
$$

After application of $\gamma^{\prime}$ to $u\left(r_{i}\right)$ as in (10) one obtains

$$
\begin{aligned}
m_{i}^{\exp } & =\gamma^{\prime} a\left(r_{i}\right)+u\left(r_{i}\right) \\
& =\left(1+\gamma^{\prime}\right) m_{i} / 2+\left(1-\gamma^{\prime}\right)\left(m_{i-1}+m_{i+1}\right) / 4 \\
& =(1-2 \gamma) m_{i}+\gamma\left(m_{i-1}+m_{i+1}\right)
\end{aligned}
$$

with $\gamma=\left(1-\gamma^{\prime}\right) / 4$. Eq. $(12 \mathrm{~d})$ is at the basis of the averaging of adjacent sites which we used previously [25]. Förster et al. even average completely over two adjacent sites $m_{i} \rightarrow\left(m_{i}+m_{i+1}\right) / 2$ [27.

Now we turn to the calculation of $\gamma^{\prime}$. Expressing the expectation value in the exponent in momentum space yields

$$
\begin{aligned}
& \frac{1}{N} \sum_{j}\left\langle\hat{\Theta}^{2}\left(\vec{r}_{j}\right)\right\rangle=\frac{1}{N} \sum_{\vec{k}}\left\langle\hat{\Theta}^{\dagger}(\vec{k}) \hat{\Theta}(\vec{k})\right\rangle \\
& =\frac{\hbar}{2 M N} \sum_{\vec{k}} \frac{1}{\omega(\vec{k})}\left(1+\frac{2}{\exp \left(\hbar \omega / k_{\mathrm{B}} T\right)-1}\right)
\end{aligned}
$$

where we used $\hat{\Theta}(\vec{k})=\sqrt{\hbar /(2 M \omega(\vec{k}))}\left(\hat{a}_{\vec{k}}+\hat{a}_{\vec{k}}^{\dagger}\right)$ with the mass $M$. The first term in the bracket in (13b) stands for the zero-point motion since it survives even for $T \rightarrow 0$. The second term in the bracket is the bosonic occupation number at finite temperature. Rearranging the exponentials yields

$$
\begin{aligned}
\gamma^{\prime} & =\exp (-D / 2) \\
D & =\frac{\hbar v}{2 M} \int \frac{1}{\omega(\vec{k})} \operatorname{coth}\left(\frac{\hbar \omega(\vec{k})}{2 k_{\mathrm{B}} T}\right) \frac{d^{3} k}{(2 \pi)^{3}}
\end{aligned}
$$

where $v$ stands for the volume per spin site.

For a comparison with experimental data we can focus on the low-temperature behaviour of (14). A close inspection of (14) reveals that $D=D_{1}+D_{2}+\mathcal{O}\left(T^{3}\right)$ where $D_{1}$ is constant and $D_{2}$ is of order $T^{2}$. With the help of the input from Ref. 29] $D_{1}$ and $D_{2}$ are determined in the appendix. One obtains

$$
\begin{aligned}
& D_{1}=\frac{(3 / \pi)^{2 / 3}}{2 \sqrt{2}} \frac{\Delta_{\text {trip }}}{u_{0} \bar{\xi}_{0} v^{2 / 3}} \\
& D_{2}=\frac{\left(k_{\mathrm{B}} T\right)^{2}}{6 \sqrt{2} \bar{\xi}_{0}^{3} u_{0} \Delta_{\text {trip }}}=\left(\frac{T}{T^{*}}\right)^{2}
\end{aligned}
$$

where $\Delta_{\text {trip }}$ is the singlet-triplet gap and $\bar{\xi}_{0}$ and $u_{0}$ a characteristic length and characteristic energy per volume, respectively, defined and given in Ref. [29]. The value of $D_{1}$ for $\mathrm{CuGeO}_{3}$ is 3.71 and the characteristic temperature $T^{*}$ is $16.9 \mathrm{~K}$.

The value of $D_{1}$ leads to a reduction of the alternating component $\gamma^{\prime}=0.16$ and the parameter $\gamma$ takes the value 0.21 . This is in very good agreement with the values 0.19 and 0.20 which we found previously by fitting theory to experiment [25].

Further support is gained from the estimate for $T^{*}$. In Fig. 11 the temperature dependence of the widths $W$ of the NMR lines are shown which are dominated by the alternating component. Hence they are expected to be proportional to $\gamma^{\prime}=\exp \left(-\left(T / T^{*}\right)^{2} / 2\right)$ to which they are compared. 


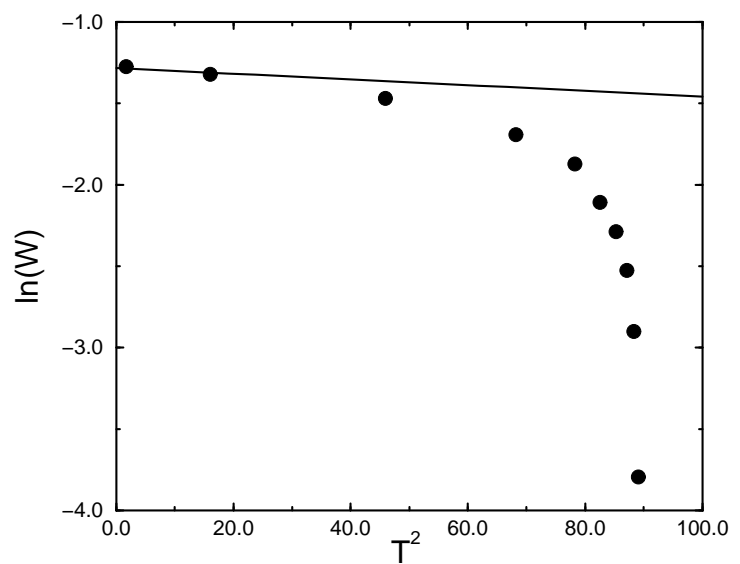

FIG. 1. Solid line: theory from eq. (15b $)$; symbols: experimental data (the error is less than a fifth of the symbol size).

The small number of data points at low $T$ does not allow for a complete quantitative test of the theoretical prediction. But the order of magnitude of the reduction of the line width at low temperatures is the correct one.

If there were an adiabatic treatment of the incommensurate phase at finite temperatures this would yield a line width which saturates exponentially at low temperatures due to the gap in the spin system [32,24]. Fig. 1 indicates clearly that there is no exponential saturation of the line width for $T \rightarrow 0$, but a behaviour $W-W(T=0) \propto-T^{2}$. This is a direct evidence for the presence of low-lying, gapless fluctuations. The quadratic behaviour in $T$ of the decrease corroborates the result (15b) and hence the existence of a three-dimensional nondegenerate dispersion.

At temperatures closer to the transition the line width is reduced much more strongly. This is due to the fluctuations in the spin system itself which are not within the scope of the present treatment.

In spite of the crudeness of the present estimates the agreement gives evidence that the basic idea, phasonic fluctuations leading to an average of the local magnetizations, is correct.

\section{NUMERICAL RESULTS}

Here we present some numerical results obtained by DMRG. The calculation treats the phonons adiabatically, i.e. the local distortions $\delta_{i}$ are real numbers which are found by minimizing the ground state energy of the following Hamiltonian 26,24,

$$
H=\sum_{i}\left[J\left(\left(1+\delta_{i}\right) \mathbf{S}_{i} \mathbf{S}_{i+1}+\alpha \mathbf{S}_{i} \mathbf{S}_{i+2}\right)+\frac{K}{2} \delta_{i}^{2}-h S_{i}^{z}\right] .
$$

The $\delta_{i}$ are determined self-consistently [26,24. Numerically, this is achieved by iteration. Since it is found that an array of equidistant solitons represents the energetically most favorable configuration [25,24] we use as initial distortion $\delta_{i} \propto \cos \left(q r_{i}\right)$ with $q=\pi+2 \pi m, m$ being the average magnetization or $\delta_{i} \propto \operatorname{sign}\left(\cos \left(q r_{i}\right)\right)$. After about 10 iterations, one reaches a stable distortion pattern. This pattern shows no discernible differences depending on which start configuration has been used.

For the calculation of the lowest state with $S=1$ for a given modulation we use the finite size algorithm 33, 34. In each iteration we keep $m=64$ states. Periodic boundary conditions are applied. Keeping $m=128$ states leads to a change of the calculated energy of the order of $10^{-5}$. For large chain lengths and large dimerizations the energy change due to a change of the distance by a few sites between two neighboring solitons is very small. This is due to the exponential localization of the solitons. So care has to be taken to avoid spurious shifts which hinder the following fit analysis.

In Fig. 2 the local distortions and the local magnetizations are shown as they are found after the procedure described above. The solid lines are fits of the following form

$$
\begin{aligned}
m_{i}=\frac{W}{2}\left\{\frac{1}{R}\right. & \operatorname{dn}\left(\frac{r_{i}}{k_{\mathrm{m}} \xi_{\mathrm{m}}}, k_{\mathrm{m}}\right)+ \\
& \left.+(-1)^{i} \operatorname{cn}\left(\frac{r_{i}}{k_{\mathrm{m}} \xi_{\mathrm{m}}}, k_{\mathrm{m}}\right)\right\} \\
\delta_{i}= & \delta \operatorname{sn}\left(\frac{r_{i}}{k_{\mathrm{d}} \xi_{\mathrm{d}}}, k_{\mathrm{d}}\right),
\end{aligned}
$$

where the parameters $W, R, \delta, k_{\mathrm{m}}$, and $k_{\mathrm{d}}$ are taken to be the fit parameters. The periodicity $L$ of the solitons is fixed by the average magnetization $m=1 / L$ which results in the relation defining the soliton widths $\xi_{\mathrm{m}}$ and $\xi_{\mathrm{d}}$ as a function of $L$ and $k_{\mathrm{m} / \mathrm{d}}$

$$
4 m k_{\mathrm{m} / \mathrm{d}} K\left(k_{\mathrm{m} / \mathrm{d}}\right) \xi_{\mathrm{m} / \mathrm{d}}=1 \text {. }
$$

Another relation can be deduced from the average of the dn-function which is $\pi /(2 K(k))$ [35] which in the continuum limit is related to the average magnetization. One obtains

$$
m=\frac{\pi W}{4 R K\left(k_{\mathrm{m}}\right)},
$$

a relation which was well fulfilled (within 1 to $4 \%$ ) by our fit parameters.

The motivation for the equations (17) is twofold. First, such fits are used to describe the experimental data 1921 . Second, the continuum approaches provide results of the above kind [2, 3, 7, 6, 12,22. The continuum results predict that the magnetic and the distortive parameters are identical

$$
k_{\mathrm{m}}=k_{\mathrm{d}} \Leftrightarrow \xi_{\mathrm{m}}=\xi_{\mathrm{d}} .
$$

The DMRG results can be fitted very well by (17). In this respect, the continuum approach works fine. But in other respects it fails. 


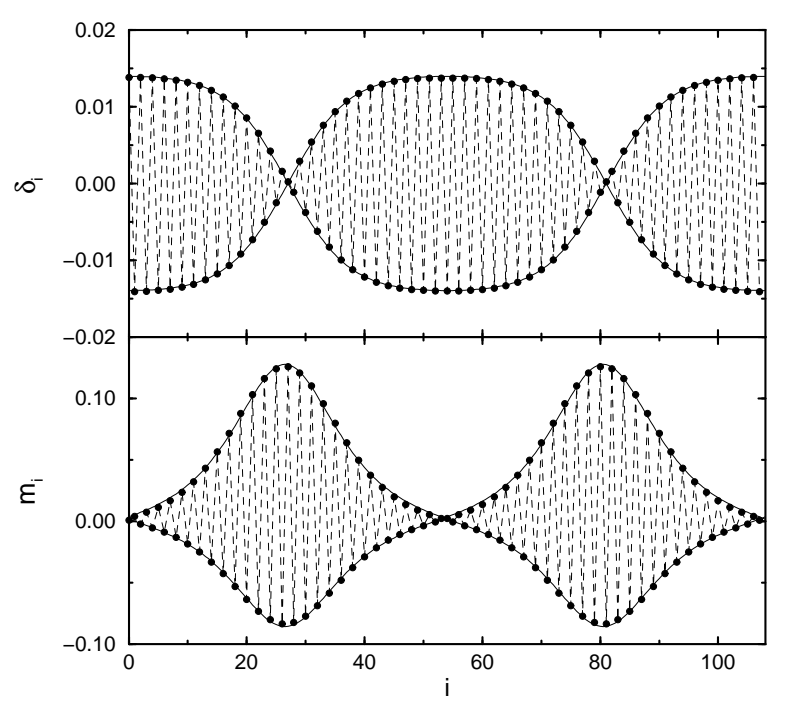

FIG. 2. Upper panel: local distortions. Symbols stand for the self-consistent DMRG-result at $K=18 J$ and $\alpha=0.35$; solid line stems from (17b) with $\delta=0.014, k_{\mathrm{d}}=0.959$, and $\xi_{\mathrm{d}}=10.5$. Lower panel: local magnetizations; the solid line stems from (17a) with $W=0.21, R=5.0, k_{\mathrm{m}}=0.992$, and $\xi_{\mathrm{m}}=7.9$.

Taking, for instance, the results for the amplitudes of the non-alternating and the alternating component [4.,22] we get for $W$ and $R$

$$
\begin{aligned}
& W=\sqrt{\frac{2 \Delta_{\text {trip }}}{\pi v_{\mathrm{S}}}} \\
& R=k \sqrt{\frac{2 \pi v_{\mathrm{S}}}{\Delta_{\text {trip }}}}
\end{aligned}
$$

Inserting some reasonable number for $\mathrm{CuGeO}_{3} \Delta_{\text {trip }}=$ $24 K, k \approx 1, v_{\mathrm{S}}=\frac{\pi}{2} J(1-1.12 \alpha$ ) [36] with $\alpha=0.35, J=$ $160 K$ yields $W=0.32$ and $R=6.3$. So the agreement with the numerical results presented in Fig. 2 is not good, but the right order of magnitude is reproduced. Another indication that eqs. (21) have to be extended is the fact that in a $1 \mathrm{D}$ approach for $\alpha>\alpha_{c}=0.2142$ a gap opens and $v_{\mathrm{S}}$ is no longer well defined.

Similar to the amplitudes, the unique soliton width is given in the framework of the present continuum theories by

$$
\xi=v_{\mathrm{S}} / \Delta_{\text {trip }}
$$

which takes the value 6.4 for the above numbers. Again, this is too low compared to our numerical results.

The most important discrepancy is the difference between the magnetic soliton width $\xi_{\mathrm{m}}=7.9$ and the distortive soliton width $\xi_{\mathrm{d}}=10.5$. Note the ratio $\xi_{\mathrm{d}} / \xi_{\mathrm{m}}=$ 1.33. This is very interesting because such a ratio can explain the different experimental findings for the soliton width. By X-ray measurement [17], $\xi_{\mathrm{d}}$ was determined to be $13.6 \pm 0.3$ whereas by NMR $\xi_{\mathrm{m}}$ was found to vary between about 10 and 6 with the higher number close to the transition. In fair accordance with our calculation, the X-ray measurement (susceptible to the distortion) yields a value about 1.4 larger than the value obtained by NMR [21] (susceptible to the local magnetizations). We will elucidate this issue further at the end of the following section.

\section{COMPARISON TO EXPERIMENTAL DATA}

In this section we attempt a quantitative comparison to the experimental results obtained recently in high quality 21]. We will do this on the basis of the Hamiltonian (16), i.e. a magnetic one dimensional Hamiltonian with adiabatic phonon treatment. We use $\alpha=0.35$ and $K=18 \mathrm{~J}$. The value of frustration results from fits of the temperature dependence of the susceptibility 37,38. The value of $K$ is then necessary to account for the amount of dimerization $\delta \approx 0.014$ which yields the correct size of the singlet-triplet gap in $\mathrm{CuGeO}_{3}$ of $\Delta_{\text {trip }} / J=24 K / 160 K=0.15$. This value of $K$ provides also a reasonable estimate for the critical magnetic field at $T=0$ [39]. One must be aware, however, that the inclusion of higher dimensional magnetic couplings implies a considerably larger dimerization to keep the same gap [40]. A hint that the actual $K$ value could be smaller is provided by the adiabatic analysis of the spin-Peierls temperature $T_{\mathrm{SP}}$. There a value of $K \approx 11$ had to be used to reproduce $T_{\mathrm{SP}} \approx 14.4$ [32]. We will discuss the effects of the neglect of the higher dimensional couplings in the concluding section.

In Fig. 3 the amplitudes computed numerically (filled circles) as defined in (17a) are contrasted to the experimental ones (filled squares; after 21]). To obtain the computed values the self-consistently determined patterns are least-square fitted with the functions given in $(17 \mathrm{a})$. The least square fits are very $\operatorname{good}\left(\chi^{2} \approx\right.$ $\left.10^{-3}-10^{-4}\right)$, but not excellent. There are tiny deviations at the magnetic tails of the solitons, see Fig. 2 . The fits for the lattice distortions on the basis of (17b) are better by two orders of magnitude. No deviations are discernible. 


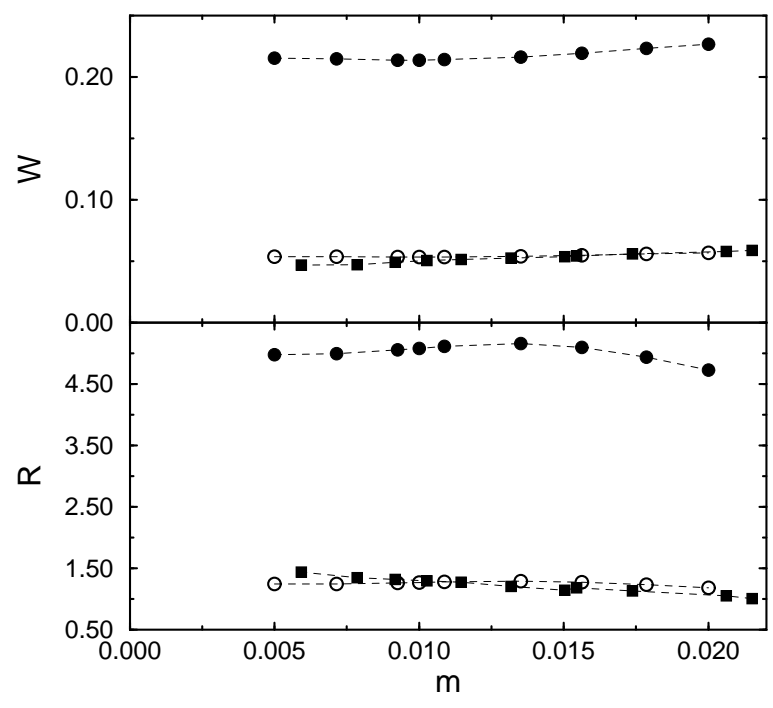

FIG. 3. Amplitudes as defined in eq. (17a). Filled circles: DMRG calculation for $\alpha=0.35$ and $K=18$ (corresponding to $\delta=0.014$ ); open circles: by $\gamma^{\prime}=0.25$ renormalized DMRG data. Filled squares: experimental NMR data after Ref. [21].

For $W$ and $R$, the striking difference between experiment and theory can be remedied by using a factor of $\gamma^{\prime}=0.25$. The theoretical results renormalized in this way are shown as open circles in Fig. 3. The simultaneous reduction of $W$ and of $R$ leaves the homogeneous part $u\left(r_{i}\right)$ of the local magnetization $m_{i}$ unchanged but reduces the alternating part $a\left(r_{i}\right)$. This is exactly what we proposed in section II to be the effect of phasonic zero-point motion. The agreement between renormalized theory and experiment is good and provides further support for the phasonic averaging.

In Fig. 1 the results for the distortive and the magnetic soliton widths are plotted. It is obvious that they are not the same as was assumed hitherto. Their ratio is to very good approximation constant for small magnetizations but grows for larger $m$. This aspect is in qualitative agreement with the experimental situation where there is also a striking difference between the $\mathrm{X}$-ray result (distortion) and the NMR result (local magnetizations). The fact that the agreement is quantitatively not better can be attributed to several circumstances. The analysis of the X-ray data [17] was done on the assumption of constant soliton widths which is not justified. Furthermore, our theoretical analysis is still based on a one-dimensional model only. It is also obvious that the qualitative evolution of the magnetic soliton width is not yet understood. Experimentally, it decreases with increasing magnetization whereas it increases in our computation.

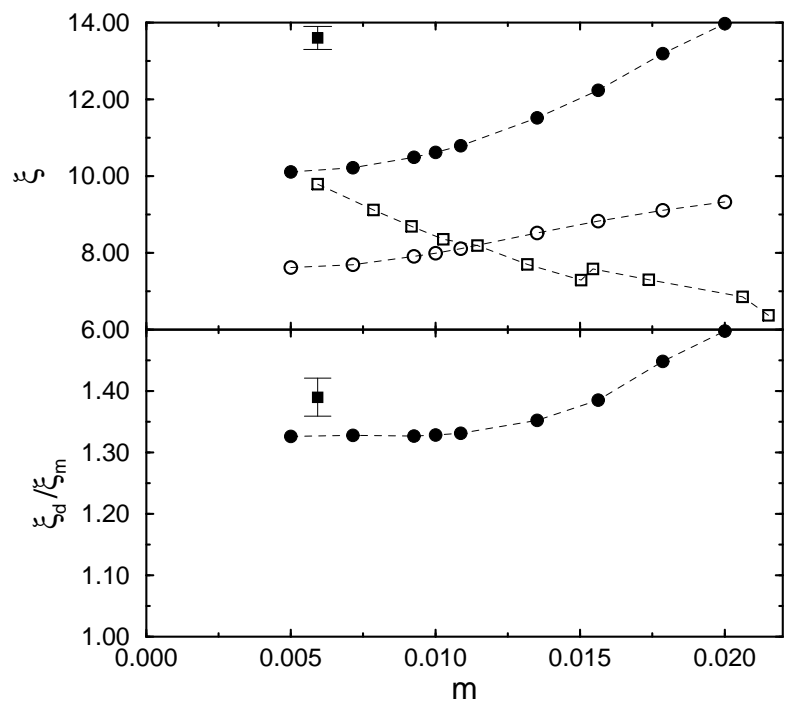

FIG. 4. Soliton widths: $\xi_{\mathrm{d}}$ distortive; $\xi_{\mathrm{m}}$ magnetic, see eq. (17). Circles: DMRG results; Open squares: experimental NMR data after Ref. 21, filled square: value proposed in Ref. [17]. Upper panel: filled symbols are based on the distortion; open symbols are based on the local magnetizations $m_{i}$. Lower panel: ratios.

Based on the lower panel of Fig. A we conclude that the ratio $\xi_{\mathrm{d}} / \xi_{\mathrm{m}}$ becomes constant if the solitons are sufficiently separated. Hence the ratio does not depend on $m$ or $K$ as long as $1 / m$ is large enough compared to $\xi_{\mathrm{d}}$. Recall that solitons are exponentially localized objects. The amplitudes and spatial shapes do not depend on the overall energy scale $J$ either. Thus the only parameter left is the frustration $\alpha$. So we are led to an analysis of the ratio $\xi_{\mathrm{d}} / \xi_{\mathrm{m}}$ as function of $\alpha$. The results are depicted in Fig. 5 .

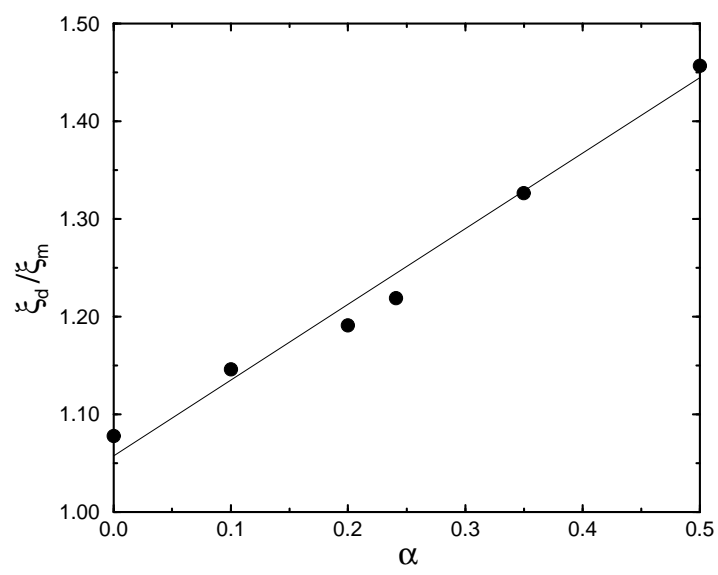

FIG. 5. Dependence of the ratio $\xi_{\mathrm{d}} / \xi_{\mathrm{m}}$ on the frustration $\alpha$. The values $K$ are chosen such that the $\xi_{\text {d }}$ ranges between 5 and 10 , i.e. not too small but always way below the sample length of $L=108$. The values $(\alpha, K)$ are $(0,3),(0.1,4),(0.2,6.2),(0.241,8),(0.35,18),(0.5,37)$. Solid line: regression with intercept 1.06 and slope 0.77 . 
It is evident that the frustration is the important control parameter for the ratio of $\xi_{\mathrm{d}}$ and $\xi_{\mathrm{m}}$. The dependence seems to be roughly linear. It should be noted that even at zero frustration the two soliton widths are not identical. The experimental finding of a relatively large values of $\xi_{\mathrm{d}} / \xi_{\mathrm{m}}$ is a strong indication for an important frustration in agreement with the $\chi(T)$ fits 37,38. A quantitative analysis appears presently premature since the effects of higher dimensional couplings are not known yet.

The deviation in the amplitudes as given in eqs. (21) as well as the non-equality of the soliton widths suggests an extension of the existing continuum theories. The fact that the non-equality is strongly enhanced by the frustration points clearly into the direction that the backscattering cosine terms in the sine-Gordon models [5,22] is the origin of this effect. Analysing the self-consistent harmonic treatment of Nakano and Fukuyama [4,5] reveals one aspect where a difference between the magnetic and the distortive soliton can appear. This is the so far neglected spatial dependence of the renormalizing factors $\exp \left(-\left\langle\hat{\Theta}^{2}\right\rangle / 2\right)$ and $\exp \left(-2\left\langle\hat{\Theta}^{2}\right\rangle\right)$, respectively. (Note that this operator $\hat{\Theta}$ is not related to phasons of distortive origin. But it stands for gaussian magnetic fluctuations and their formal treatment bears many analogies to the treatment of phasons.) Further work along these lines is in progress.

\section{DISCUSSION}

In this work we calculated the effect of phasonic zeropoint motion on the local magnetizations $m_{i}$. This nonadiabatic effect can be viewed as virtual oscillations about the static situation. It leads to a reduction of the alternating component of the $m_{i}$. The reduction factor $\gamma^{\prime}$ as in eq. (9) is similar to a Debye-Waller factor which is induced by phononic motion.

By the inclusion of the nonadiabatic phason motion we could explain the so far not understood difference between experimental NMR line shapes 19 21] and previous theoretical predictions $13,25,27,23$. . Hence the reduction of the NMR line width compared to the adiabatically predicted one is a strong evidence for the importance of nonadiabaticity in $\mathrm{CuGeO}_{3}$. Nonadiabaticity is also considered to be important for temperature dependent couplings and induced frustration (cf. 41 43]).

The second main point of our work is the difference between the distortive and the magnetic soliton width. To our knowledge, such a difference has so far not been reported in the literature. But it helps to understand the differing X-ray and NMR results. The ratio $\xi_{\mathrm{d}} / \xi_{\mathrm{m}}$ is controlled by the frustration $\alpha$. It is fairly close but not equal to unity at zero frustration and grows roughly linear to almost 1.5 at the Majumdar-Ghosh point $\alpha=$ $1 / 2$. Thus, further evidence for a sizable frustration in $\mathrm{CuGeO}_{3}$ is provided.
Aiming for quantitative agreement with experiment some questions remain. Choosing a small dimerization (the corresponding large $K$, respectively), which reproduces the correct gap value and critical magnetic field in a one-dimensional model, leads to amplitudes $W$ and $R$ which have to be renormalized by about $\gamma^{\prime}=0.25$ and not by $\gamma^{\prime}=0.16$ as estimated in section II. Thus these amplitudes seem to be too small. The magnetic soliton width $\xi_{\mathrm{m}}$, is too low by about $20 \%$.

Choosing, however, a larger dimerization (the corresponding small $K$, respectively), which reproduces the gap value resulting from averaging the dispersion $\omega(\vec{k})$ perpendicular to the chains along $k_{\mathrm{b}}$ [40], leads to larger amplitudes $W$ and $R$. These larger amplitudes can be nicely reconciled with the experimental findings by a value very close to $\gamma^{\prime}=0.16$. This is essentially what was done in the previous work [25]. The argument in favor of the larger gap is that the neglect of the interchain coupling would lead to this larger gap [40]. But the corresponding soliton widths would be even lower (see eq. (22)) and hence in worse agreement with experiment. We conclude from this that it is not just a question of finetuning the constants to achieve agreement. The purely $d=1$ model is not sufficient.

The magnetic interchain couplings have to be taken into account as has become already apparent from the two ways to choose the relevant gap. The magnetic interchain coupling has the two following effects. First, it reduces the observed gap value for a given dimerization 25]. Thus a larger dimerization (smaller $K$ ) should be used. This is consistent with a number of recent investigations 44,45. The larger gap will lead to larger amplitudes. Second, Zang et al. 22] argued that the interchain coupling enhances the soliton widths which is exactly what is needed to reconcile theory and experiment. This enhancement is qualitatively easy to understand since an interchain coupling will favor the appearance of staggered magnetization. Regions of staggered magnetization are those were the solitons are, see Fig. 2. So these regions are extended which implies an enhancement of the soliton width. Hence, we come to the conclusion that a larger dimerization (smaller $K$ ) plus an appropriate treatment of the interchain couplings should yield results in quantitative agreement with experiment. There are also preliminary results indicating that the inclusion of interchain coupling changes the increase of $\xi_{\mathrm{m}}$ as function of $m$ to a decrease which is what is experimentally seen (cf. Fig. 4).

Summarizing, two main points are shown in the present work. The first is the importance of the phasonic fluctuations providing a natural explanation for the large amplitude differences between theory and experiment. The second is the difference between distortive $\left(\xi_{\mathrm{d}}\right)$ and magnetic $\left(\xi_{\mathrm{m}}\right)$ soliton width leading to a ratio $\xi_{\mathrm{d}} / \xi_{\mathrm{m}}$ between 1.05 and 1.45 depending on frustration. Further work to include interchain coupling is called for.

We gratefully acknowledge useful discussions with C. 
Berthier and D. Förster and the support of this work by E. Müller-Hartmann. Part of this work (GSU,FS) was supported by the SFB 341 of the DFG.

[1] J. W. Bray, L. V. Interrante, I. C. Jacobs, and J. C. Bonner, in Extended Linear Chain Compounds, edited by J. S. Miller (Plenum Press, New York, 1983), Vol. 3, p. 353 .

[2] S. A. Brazovskiǐ, Sov. Phys. JETP 51, 342 (1980).

[3] S. A. Brazovskiǐ, S. A. Gordyunin, and N. N. Kirova, JETP Lett. 31, 456 (1980).

[4] T. Nakano and H. Fukuyama, J. Phys. Soc. Jpn. 49, 1679 (1980).

[5] T. Nakano and H. Fukuyama, J. Phys. Soc. Jpn. 50, 2489 (1981).

[6] J. Mertsching and H. J. Fischbeck, Phys. Stat. Sol. (b) 103, 783 (1981).

[7] B. Horovitz, Phys. Rev. Lett. 46, 742 (1981).

[8] B. Horovitz, Phys. Rev. B 35, 734 (1987).

[9] A. I. Buzdin and V. V. Tugushev, Sov. Phys. JETP 58, 428 (1983).

[10] A. I. Buzdin, M. L. Kulić, and V. V. Tugushev, Solid State Commun. 48, 483 (1983).

[11] M. Fujita and K. Machida, J. Phys. Soc. Jpn. 53, 4395 (1984).

[12] M. Fujita and K. Machida, J. Phys. C21, 5813 (1988).

[13] T. W. Hijmans, H. B. Brom, and L. J. de Jongh, Phys. Rev. Lett. 54, 1714 (1985).

[14] M. Hase, I. Terasaki, and K. Uchinokura, Phys. Rev. Lett. 70, 3651 (1993).

[15] J. P. Boucher and L. P. Regnault, J. Phys. I France 6, 1939 (1996).

[16] V. Kiryukhin and B. Keimer, Phys. Rev. B 52, 704 (1995).

[17] V. Kiryukhin, B. Keimer, J. P. Hill, and A. Vigliante, Phys. Rev. Lett. 76, 4608 (1996).

[18] V. Kiryukhin et al., Phys. Rev. B 54, 7269 (1996).

[19] Y. Fagot-Revurat et al., Phys. Rev. Lett. 77, 1861 (1996).

[20] M. Horvatić et al., Physica B246-247, 22 (1998).

[21] M. Horvatić et al., condmat/9812370.

[22] J. Zang, S. Chakravarty, and A. R. Bishop, Phys. Rev. B 55, R14705 (1997).

[23] G. S. Uhrig, F. Schönfeld, M. Laukamp, and E. Dagotto, Eur. Phys. J. B 7, 67 (1999).

[24] F. Schönfeld, G. Bouzerar, G. S. Uhrig, and E. MüllerHartmann, Eur. Phys. J. B 5, 521 (1998).

[25] G. S. Uhrig, F. Schönfeld, and J. Boucher, Europhys. Lett. 41, 431 (1998).

[26] A. E. Feiguin, J. A. Riera, A. Dobry, and H. A. Ceccatto, Phys. Rev. B 56, 14607 (1997).

[27] D. Förster, Y. Meurdesoif, and B. Malet, condmat/9802245.

[28] R. H. McKenzie and J. W. Wilkins, Phys. Rev. Lett. 69, 1085 (1992).

[29] S. M. Bhattacharjee, T. Nattermann, and C. Ron- newinkel, Phys. Rev. B 58, 2658 (1998).

[30] T. Lorenz, U. Ammerahl, R. Ziemes, and B. Büchner, Phys. Rev. B 54, R15610 (1996).

[31] M. Braden et al., Phys. Rev. B 54, 1105 (1996).

[32] A. Klümper, R. Raupach, and F. Schönfeld, Phys. Rev. B 59, 3612 (1999).

[33] S. R. White, Phys. Rev. Lett. 69, 2863 (1992).

[34] S. R. White, Phys. Rev. B 48, 10345 (1993).

[35] M. Abramowitz and I. A. Stegun, Handbook of Mathematical Functions (Dover Publisher, New York, 1964).

[36] A. Fledderjohann and C. Gros, Europhys. Lett. 37, 189 (1997).

[37] J. Riera and A. Dobry, Phys. Rev. B 51, 16098 (1995).

[38] K. Fabricius et al., Phys. Rev. B 57, 1102 (1998).

[39] T. Lorenz et al., Phys. Rev. Lett. 81, 148 (1998).

[40] G. S. Uhrig, Phys. Rev. Lett. 79, 163 (1997).

[41] G. S. Uhrig, Phys. Rev. B 57, R14004 (1998).

[42] G. Wellein, H. Fehske, and A. P. Kampf, Phys. Rev. Lett. 81, 3956 (1998).

[43] A. Weiße, G. Wellein, and H. Fehske, condmat/9901262.

[44] B. Büchner, H. Fehske, A. P. Kampf, and G. Wellein, condmat/9806022.

[45] R. Werner, C. Gros, and M. Braden, condmat/9810038.

[46] N. W. Ashcroft and N. D. Mermin, Solid State Physics (Saunders College, Philadelphia, 1976).

\section{APPENDIX:}

Based on eq. (14b) and Ref. [29] we present here estimates for the two leading contributions $D_{1}$ and $D_{2}$. Focussing on the low-lying excitations we adopt (11) even though we do not know whether at larger values of $\vec{k}$ the dispersion is still described by (11).

The mass density $\rho$ is proportional to $M / v$. If each spin site moved in the same way on shifting the modulation $M=v \rho$ were reasonable. Since, however, the modulation does not have the same amplitude for all sites an effective reduction has to be taken into account. For sinusoidal modulation this factor is $1 / 2$, i.e. $M=v \rho / 2$, due to the average value of $\sin ^{2}$. For all other modulations the reduction factor will be between $1 / 2$ and 1 ; we choose $1 / 2$ since the higher harmonic content of the modulation is very small [17].

With $k_{x} \rightarrow k_{x} \sqrt{\rho / c_{x}}, k_{y} \rightarrow k_{y} \sqrt{\rho / c_{y}}$, and $k_{z} \rightarrow$ $k_{z} \sqrt{\rho /\left|2 c_{z}\right|}$ as well as $c_{i}=r_{0} T_{0} \xi_{0 i}^{2}$ and $\bar{\xi}_{0}=$ $\left(\xi_{0 x} \xi_{0 y} \xi_{0 z}\right)^{1 / 3}$ we obtain

$$
\begin{aligned}
D & =\frac{\hbar}{\rho} \frac{\rho^{3 / 2}}{\sqrt{2 c_{x} c_{y} c_{z}}} \int \frac{d^{3} k}{(2 \pi)^{3}} \frac{1}{k} \operatorname{coth}\left(\hbar k /\left(2 k_{\mathrm{B}} T\right)\right) \\
& =\frac{\hbar}{\rho \sqrt{2}}\left(\frac{\rho}{\bar{\xi}_{0}^{2} r_{0} T_{0}}\right)^{3 / 2} \int \frac{d^{3} k}{(2 \pi)^{3}} \frac{1}{k} \operatorname{coth}\left(\frac{\hbar k}{2 k_{\mathrm{B}} T}\right)
\end{aligned}
$$

For the value of $u_{0}=r_{0} T_{0}$ and other values see Ref. [29]. We split $D$ in the zero temperature contribution $D_{1}$ and the temperature dependent rest $D_{2}$ by using $\operatorname{coth}(x / 2)=$ $1+2 /(\exp (x)-1)$. 
We estimate $D_{1}$, the zero temperature reduction due to the zero-point motion of the phasons. It is difficult to compute $D_{1}$ reliably since, in principle, information of all the phasons is required, not only the lowest lying ones. But an estimate which determines the order of magnitude is possible by using an upper cutoff $k_{\max }$ as in the Debye model of phonons [46]. We start with

$$
\begin{aligned}
D_{1} & =\frac{\hbar}{\rho} \int \frac{d^{3} k}{(2 \pi)^{3}} \frac{1}{\omega(\vec{k})} \\
& =\frac{\hbar \sqrt{\rho}}{2 \sqrt{2} \pi^{2}\left(\bar{\xi}_{0} \sqrt{u_{0}}\right)^{3}} \int_{0}^{k_{\max }} k d k \\
& =\frac{\hbar \sqrt{\rho}}{4 \sqrt{2} \pi^{2}\left(\bar{\xi}_{0} \sqrt{u_{0}}\right)^{3}} k_{\max }^{2} .
\end{aligned}
$$

The value for $k_{\max }$ after rescaling is obtained from the number of possible phasonic excitations

$$
\begin{aligned}
1 & =v \int \frac{d^{3} k}{(2 \pi)^{3}} \\
& =\left(\frac{\rho}{\bar{\xi}_{0}^{2} u_{0}}\right)^{3 / 2} \frac{v}{2 \sqrt{2} \pi^{2}} \int_{0}^{k_{\max }} k^{2} d k \\
\Rightarrow k_{\max } & =\sqrt{\frac{u_{0}}{\rho} \bar{\xi}_{0}}\left(\frac{6 \sqrt{2} \pi^{2}}{v}\right)^{1 / 3} .
\end{aligned}
$$

Eqs. (A20 A3d) and the singlet-triplet gap $\Delta_{\text {trip }}=$ $\hbar \sqrt{u_{0} / \rho}$ [29 together lead finally to (15a). It should be noted that the volume per spin site can be found from the lattice constants by $v=a b c / 2$ with $a=4.79 \cdot 10^{-10} \mathrm{~m}$, $b=8.40 \cdot 10^{-10} \mathrm{~m}$, and $c=2.94 \cdot 10^{-10} \mathrm{~m}$ [31]. The division by 2 is necessary since there are two $\mathrm{Cu}$ ions in each unit cell.

Next we compute $D_{2}$ where we are only interested in the leading temperature dependence. Thus the result depends only on the lowest lying excitations and the assumption of a dispersion as (1) is well justified. We find

$$
\begin{aligned}
D_{2} & =\frac{\hbar}{\sqrt{2} \pi^{2} \rho}\left(\frac{\rho}{\bar{\xi}_{0}^{2} u_{0}}\right)^{3 / 2} \int_{0}^{\infty}\left(\exp \left(\frac{\hbar k}{k_{\mathrm{B}} T}\right)-1\right)^{-1} k d k \\
& =\frac{\left(k_{\mathrm{B}} T\right)^{2}}{\sqrt{2} \pi^{2} \hbar \rho}\left(\frac{\rho}{\bar{\xi}_{0}^{2} u_{0}}\right)^{3 / 2} \int_{0}^{\infty}(\exp (k)-1)^{-1} k d k \\
& =\frac{\sqrt{\rho}\left(k_{\mathrm{B}} T\right)^{2}}{6 \sqrt{2} \hbar \bar{\xi}_{0}^{3} u_{0}^{3 / 2}}=\frac{\left(k_{\mathrm{B}} T\right)^{2}}{6 \sqrt{2} \bar{\xi}_{0}^{3} u_{0} \Delta_{\text {trip }}} \\
& =\left(\frac{T}{T^{*}}\right)^{2} .
\end{aligned}
$$

With the numbers $\Delta_{\text {trip }}=24 \mathrm{~K}, u_{0}=650 \mathrm{~mJ} / \mathrm{cm}^{3}$ and $\bar{\xi}_{0}=0.31 \mathrm{~nm}$ the characteristic temperature $T^{*}$ can be estimated to be $16.9 \mathrm{~K}$. 